\title{
3 Research Square \\ Use of Elamipretide in Patients Assigned Treatment in the Compassionate Use Program: Case Series in Four Rare Orphan Diseases
}

Mary Kay Koenig ( $\square$ Mary.K.Koenig@uth.tmc.edu )

University of Texas John P and Katherine G McGovern Medical School https://orcid.org/0000-00015126-8515

\section{S. Nick Russo}

University of Texas McGovern Medical School: The University of Texas Health Science Center at Houston John P and Katherine G McGovern Medical School

\section{Kim L. McBride}

Nationwide Children's Hospital

Hans Tomas Bjornsson

Landspítali University Hospital: Landspitali

\section{Brynja Bjork Gunnarsdottir}

Landspítali University Hospital: Landspitali

\section{Amy Goldstein}

Childrens Hospital of Philadelphia

\section{Scott A. Falk}

University of Pennsylvania Perelman School of Medicine

\section{Research}

Keywords: Mitochondrial Disease, Elamipretide, Cardiolipin, MEGDEL, Barth syndrome, Sengers syndrome Posted Date: October 12th, 2021

DOl: https://doi.org/10.21203/rs.3.rs-927266/v1

License: (c) (i) This work is licensed under a Creative Commons Attribution 4.0 International License. Read Full License 


\section{Abstract}

\section{Background:}

Several mitochondrial diseases are caused by pathogenic variants that impair membrane phospholipid remodeling. There are no US Food and Drug Administration (FDA)-approved therapies for these conditions. Elamipretide is a cell-permeable peptide that has been shown to target the inner mitochondrial membrane where it binds to cardiolipin, resulting in improved membrane stability, cellular respiration, and ATP production. Elamipretide has been evaluated in adult and adolescent patients with mitochondrial disorders such as primary mitochondrial myopathy and Barth syndrome, producing clinical and functional improvements. There is little experience with elamipretide in younger patients and those with other mitochondrial diseases.

\section{Results:}

We describe the use of elamipretide in a series of patients with various mitochondrial disorders providing insight regarding dosing of the drug for these patients, especially dosing in young patients.

\section{Conclusions:}

Based on our experience, a dose of approximately $0.5 \mathrm{mg} / \mathrm{kg} / \mathrm{day}$ is well tolerated in pediatric patients aged $<12$ years.

\section{Background}

Several mitochondrial diseases are caused by pathogenic variants that impair membrane phospholipid remodeling. These include Barth syndrome (MIM \#302060), 3-methylglutaconic aciduria, deafness, encephalopathy, Leigh-like disease (MEGDEL, MIM \#614739), and Sengers syndrome (MIM \#212350). Many genetically linked mitochondrial diseases result in dilated or hypertrophic cardiomyopathies. There are no US Food and Drug Administration (FDA)-approved therapies for these conditions. Elamipretide is a cell-permeable peptide that has been shown to target the inner mitochondrial membrane where it binds to cardiolipin, resulting in improved membrane stability, cellular respiration, and ATP production. ${ }^{1,2}$ Elamipretide has been evaluated in adult and adolescent patients with mitochondrial disorders such as primary mitochondrial myopathy and Barth syndrome, producing clinical and functional improvements. 3,4 There is little experience with elamipretide in younger patients and those with other mitochondrial diseases. However, the FDA has provided guidance on expanded access for the use of investigational drugs for patients with serious diseases who lack therapeutic alternatives when there is a biologic plausibility to predict efficacy. ${ }^{5}$ We describe the use of elamipretide in a series of patients with various mitochondrial disorders with a goal of providing insight on the dosing and rationale for the use of the drug.

\section{Results/case Series}




\section{Case 1: Barth syndrome}

A male who presented with heart gallop within hours of birth was found to have lactic acidosis and severe left ventricular (LV) dysfunction characterized by severe dilated cardiomyopathy and an ejection fraction (EF) of approximately $20 \%$. The cardiomyopathy was unresponsive to medical management. Exome sequencing identified a pathogenic variant in Tafazzin (TAZ), consistent with a diagnosis of Barth syndrome (diagnoses based on entry in a previous study at 3 weeks of age that included rapid genome sequencing) and related cardiomyopathy. It was decided to initiate elamipretide at 3 weeks of age as his EF had not significantly improved on maximal medical therapy that included milrinone, sacubitril/valsartan, and carvedilol. The initial dose of elamipretide was $0.25 \mathrm{mg} / \mathrm{kg}$ administered intravenously with weight-based upward titration to $0.5 \mathrm{mg} / \mathrm{kg}$ beginning at 1 week. Each dose was administered over 2 hours. At 4 weeks, the patient was switched to subcutaneous (SQ) dosing at 0.5 $\mathrm{mg} / \mathrm{kg} /$ day $(10 \mathrm{mg} / \mathrm{mL}$ formulation of elamipretide) and discharged from the hospital on that dose. Additional medications since discharge included filgrastim, arginine, sacubitril/valsartan, carvedilol, and spironolactone. This dosing schema resulted in a gradual improvement of the EF to between $45 \%$ and $55 \%$ although dyskinesia of the LV remained (Table 1). Neutropenia was improved on filgrastim, with normal absolute neutrophil counts ranging from 1020 to $1200 / \mathrm{mm}^{3}$ after therapy was started. The hepatic enzymes AST, ALT, and CK were normal after 1 month of age, although there was a mild persistent lactic acidosis ranging from 2.0 to $4.4 \mathrm{mmol} / \mathrm{L}$. At 4 months of age, the patient was meeting all developmental milestones. There were no adverse events related to elamipretide, regardless of the route of administration (IV or SC). Unfortunately, the patient died at 5.5 months of age as a result of his underlying disease, with a normal appearing heart on autopsy. The total time on treatment with elamipretide for this patient was 150 days.

Table 1. Barth Syndrome patient (Patient Case 1) imaging data 


\begin{tabular}{|llccllll|}
\hline Date & Modality & SF\% & EF\% & $\begin{array}{l}\text { LVEDDmm } \\
(\mathbf{Z})\end{array}$ & $\begin{array}{l}\text { LV } \\
\text { Hypertrophy }\end{array}$ & $\begin{array}{l}\text { LV } \\
\text { Function }\end{array}$ & RV Function \\
\hline $11 / 12 / 20$ & TTE & 13 & $\begin{array}{l}26- \\
39\end{array}$ & $19(-0.1)$ & & Mod-severe & Normal \\
\hline $11 / 13 / 20$ & TTE & 21 & $\begin{array}{l}44- \\
48\end{array}$ & $19(0)$ & & $\begin{array}{l}\text { Mild, } \\
\text { dyskinetic }\end{array}$ & $\begin{array}{l}\text { Low normal to } \\
\text { mild dysfxn }\end{array}$ \\
\hline $11 / 14 / 20$ & TTE & 26 & & & Severe & $\begin{array}{l}\text { Mild, } \\
\text { dyskinetic }\end{array}$ & Mild \\
\hline $11 / 15 / 20$ & TTE & 19 & 45 & $20(+0.8)$ & & $\begin{array}{l}\text { Mild, } \\
\text { dyskinetic }\end{array}$ & Mild \\
\hline $11 / 19 / 20$ & TTE & 10 & $40-$ & $21(+1.1)$ & & $\begin{array}{l}\text { Moderate, } \\
\text { dyskinetic }\end{array}$ & Mild \\
\hline $11 / 24 / 20$ & TTE & 17 & $25-$ & $23(+2.6)$ & Moderate & $\begin{array}{l}\text { Severe, } \\
\text { dyskinetic }\end{array}$ & Normal \\
\hline $11 / 25 / 20$ & TTE & 11 & 35 & $21(+1.2)$ & Moderate & $\begin{array}{l}\text { Severe, } \\
\text { dyskinetic }\end{array}$ & Normal \\
\hline $11 / 30 / 20$ & TTE & 13 & $26-$ & $22(+1.8)$ & Moderate & $\begin{array}{l}\text { Severe, } \\
\text { dyskinetic }\end{array}$ & Normal \\
\hline
\end{tabular}

$12 / 02 / 20$ Initiation of sacubitril-valsartan

12/4/20 TTE $18 \quad 37-38 \quad 20(+0.7) \quad$ Moderate Moderate, dyskinetic Low normal

$12 / 05 / 20$ Initiation of elamipretide at $0.25 \mathrm{mg} / \mathrm{kg}$

12/7/20 TTE $15 \quad 39-66 \quad 20(-0.3) \quad$ Mild Low normal (to my eyes mild-mod) Normal

\section{$12 / 10 / 20$ Off milrinone last}

$12 / 12 / 20$ Elamipretide to $0.5 \mathrm{mg} / \mathrm{kg}$

\begin{tabular}{|llllllll|}
\hline $12 / 12 / 20$ & TTE & 22 & $41-53$ & $18(-1.0)$ & Mild & Mild-mod, dyskinetic & Normal \\
\hline $12 / 16 / 20$ & TTE & & $41-54$ & $20(+0.2)$ & Mild & Mild, dyskinetic & Normal \\
\hline $12 / 22 / 20$ & TTE & 29 & 48 & $21(+0.3)$ & Mild & Mild, dyskinetic & Normal \\
\hline $12 / 30 / 20$ & TTE & 23 & $51-54$ & $20(-0.3)$ & Mild, LVNC & Mild, dyskinetic & Normal \\
\hline $1 / 15 / 21$ & TTE & 21 & $44-46$ & $22(-0.1)$ & Mild, LVNC & Mild, dyskinetic & Normal \\
\hline $2 / 19 / 21$ & TTE & 22 & 58 & $19(-2.4)$ & Mild, LVN & Mild, dyskinetic & Normal \\
\hline $4 / 15 / 2021$ & TTE & 30 & $50-57$ & $23(-0.8)$ & Mild, LVN & Low-normal, dyskinesia & Normal \\
\hline
\end{tabular}




\section{Case 2: MEGDEL syndrome}

A male patient presented to the clinic with MEGDEL syndrome. The patient had experienced significant developmental delays in early motor development (ie, not sitting alone until 14 months or crawling until 20 months and ambulating with a walker at 3 years). Dysarthria resulted in impediments to communication that caused him to be difficult to be understood. At his best, he spoke in 4-to-5-word sentences, and his speech was approximately $50 \%$ understandable to a stranger. Although he experienced sporadic brief regressions with illness, he mostly gained skills until the age of 5 years when he began to display regression. After this age, he began to lose the ability to use his walker and by age 6 could no longer stand. Language also became progressively more dysarthric. An echocardiogram and renal evaluations at age 4 revealed no abnormalities. At 3 years of age the patient experienced an acute episode in which he was found unresponsive and had a lactic acid level of $7.9 \mathrm{mmol} / \mathrm{L}$. He received IV fluids, and symptoms resolved after an overnight hospitalization. The symptoms have not recurred. At 6 years and 7 months of age, elamipretide was initiated at a subcutaneous dose of $10 \mathrm{mg} /$ day $(0.58$ $\mathrm{mg} / \mathrm{kg} /$ day) with a predetermined plan to increase the dose to $20 \mathrm{mg} /$ day when he reached a weight of $20 \mathrm{~kg}$. Since this weight has not been attained, the patient remains on $10 \mathrm{mg}$ SQ daily at 7 years and 10 months. There have been no adverse reactions to the elamipretide, and after 1 year and 3 months of therapy at this dose, the patient has demonstrated not only a lack of continued regression but developmental progress. The patient's parents have noted improved stamina and strength. He is again speaking in 3-to-4-word sentences, and speech is more understandable. He is able to sit alone, stand with support, and is using his gait trainer.

\section{Case 3: Sengers syndrome}

This male patient presented at 3 weeks of age with severe hypertrophic cardiomyopathy, bilateral cataracts, and significant hypotonia. Whole genome analysis revealed he was homozygous for an Icelandic founder mutation (p.lle348AsnfsTer39) in the acylglycerol kinase (AGK) gene, and a diagnosis of Sengers syndrome was made based on symptoms and genotype. Genetic analysis also revealed that he was a carrier of two variants in the GPT2 gene, one from his mother [c.247C>T p $\left.\left(\operatorname{Arg} 92^{\star}\right)\right]$ and the other from his father [c.371G $>C p($ Ser124Thrl)], the latter being a variant of uncertain significance; however, his phenotype was not consistent with GPT2-deficiency, so this diagnosis was disregarded. However, it is worth noting that his presentation was unusually aggressive compared to other Icelandic patients carrying these particular founder variants, so it is not impossible that the other variants affected the phenotype in some way. The boy was initiated on beta blocker therapy which was gradually titrated up (including during the trial with elamipretide) and, beginning at age 3 months, he received elamipretide $(0.25 \mathrm{mg} / \mathrm{kg}$ IV infused over 2 hours [per protocol], every 24 hours) and at 4 months of age, it was increased to $0.50 \mathrm{mg} / \mathrm{kg}$ IV infused over 2 hours every 24 hours. This dose was then increased per weight but not otherwise modified. Therapy with elamipretide continued for approximately 6 months. The Child 
was also treated with Carnitine and Q10 supplements by neurologist. The total time on treatment with elamipretide for this patient was 187 days.

Pharmacokinetic (PK) analysis showed that elamipretide exposure was similar to that observed in other elamipretide trials. Following the initiation of therapy, the patient experienced subjective improvements from the prior week's evaluation in 13 of 21 visits using a clinical global impression scale with the global score improving from "markedly ill" to "borderline ill" during treatment (Figure 1). Cardiac improvements included an increase in the LV end-diastolic internal dimension (Z-score from -2.7 to +0.7 ), a decrease ventricular septal thickness (Z-score from 4.7 to 3.5), and a decrease in LV posterior wall thickness (Zscore from 5.8 to 4.7$)$. There were no side effects that were attributable to elamipretide. The patient experienced intermittent lactic acidosis and neutropenia that were considered secondary to his condition. After 6 months of treatment, the patient underwent an elective PEG placement after which he experienced cardiac decompensation, followed by multi-organ failure and death.

\section{Case 4: ACAD9 Deficiency}

A 29-year-old female with a history of chronic hypertrophic cardiomyopathy and lactic acidosis, identified at approximately 12 years of age (gene panel negative at that time), presented in acute cardiogenic shock from a referring hospital to the Cardiology Intensive Care Unit (ICU) with an Impella 2.5 device in place via the right axillary artery. Cardiogenic shock was triggered by lying supine in a cardiac magnetic resonance imaging machine due to worsening dyspnea on exertion (DOE) for a septal ablation procedure. The last available lab values, obtained at age 18 , were: normal CK of 33, lactate of 5.9 , pyruvate of 0.22 with L:P ratio of 27 , and normal acylcarnitine profile.

On hospital day 2, the patient went to the operating room for Impella removal and consideration for extracorporeal membrane oxygenation (ECMO) because of the large amount of hemolysis from the Impella. Patient was in shock on arrival to the operating room with a pH of 6.9 and relatively high inotropic and pressor requirements. The Impella device was removed following correction of metabolic derangements, and the patient went to the cardiothoracic surgical ICU on reasonable doses of inotropic and pressor support. Over the next several hours, the patient hemodynamically declined and was cannulated for ECMO support. The patient had persistent lactic acidosis (12 mM), renal failure, and hepatic failure from shock. Initial cardiac dysfunction was severely depressed with an estimated EF of $15-20 \%$. Other metabolic laboratory values included highly elevated creatine kinase, elevated lactate to pyruvate ratio, elevated alanine, and organic acids in the urine.

She was started on supportive therapy for this condition, including $\mathrm{N}$-acetylcysteine, I-carnitine, and dextrose. Three days after ECMO cannulation, the patient was started on elamipretide in consideration of the severity of illness and the success of this medication in similar patient presentations. The patient was initiated on elamipretide $0.5 \mathrm{~mL} \mathrm{SC}$ daily (40-mg equivalent dose) and maintained on that dose for 90 days as an inpatient. Her estimated EF continued to improve to $25 \% 4$ days after ECMO cannulation. The patient continued to hemodynamically improve and was decannulated from ECMO 5 days after initial cannulation. Heart function continued to improve, and the patient was noted to have an EF of $44 \% 6$ days 
after ECMO cannulation. Although the hospital course was complicated by ventilator-dependent respiratory failure, renal failure, and ventilator-associated pneumonia, which led to a prolonged stay, the patient was eventually discharged to inpatient rehab on hospital day 102.

Additional diagnostic testing completed during her admission included a muscle biopsy and whole exome sequencing. Muscle biopsy was sent for mitochondrial DNA sequencing and deletions, which did not show a mitochondrial DNA pathogenic variant, but did show multiple low-level deletions of unknown significance. Mitochondrial DNA copy number in muscle was normal at 93\% (no depletion). Electron transport chain analysis unexpectedly showed a normal complex I activity of $128 \%$, with complex II at $530 \%$ (perhaps due to overcompensation). Complex III and IV were normal, as was citrate synthase. Whole exome sequencing revealed compound heterozygous variants in ACAD9 [c.1594 C>T (p.R532W) (pathogenic) and c.1646 G>A (p.R549Q) (likely pathogenic)], consistent with a diagnosis of ACAD9related disease. This entity, in its later onset form, explains very well her clinical presentation, including her lactic acidosis, cardiomyopathy, and skeletal muscle weakness. She was then started on riboflavin supplementation and continued a mitochondrial cocktail with $\mathrm{N}$-acetylcysteine, levocarnitine, and thiamine. ACAD9 is typically associated with complex I deficiency; thus, it is unclear why her activity would have been normalized, although there are reports of individuals with later-onset disease related to $A C A D 9$ with normal complex I activity. ${ }^{6}$

Elamipretide was discontinued after having been administered for 65 days, with no intent to restart due to the patient's wish to discontinue, and follow-up continued with the genetics/metabolism group at the Hospital of the University of Pennsylvania.

\section{Discussion}

These cases describe the expanded access use of elamipretide in patients with a range of diseases associated with mitochondrial dysfunction and provide insight regarding dosing of the drug for these patients, especially dosing in young patients. The patients described share several phenotypic traits related to mitochondrial dysfunction. Barth Syndrome is an X-linked genetic disease caused by mutations in TAZ, the gene that encodes for a transacylase involved in the remodeling of cardiolipin. Cardiolipin is a crucial phospholipid found in the inner membrane of mitochondria that is necessary for the proper functioning of the respiratory chain. ${ }^{7}$ Barth syndrome is characterized by cardiomyopathy, neutropenia, hypotonia, and growth abnormalities. ${ }^{8,9}$ MEGDEL is caused by biallelic pathogenic variants in the $S E R A C 1$ gene that produces mitochondrial dysfunction. ${ }^{10}$ Alterations in the gene produce changes in the acyl chain composition of cardiolipin, resulting in abnormal oxidative phosphorylation. ${ }^{10}$ Sengers syndrome is a mitochondrial disease caused by mutations in mitochondrial AGK that is required for the structural integrity of mitochondria and contributes to the synthesis of cardiolipin. The disease is characterized by hypertrophic cardiomyopathy, skeletal myopathy, and cataracts. ${ }^{11,12}$ There are also other hypertrophic cardiomyopathies that are associated with mitochondrial diseases, including ACAD9. ${ }^{13,14}$ 
Elamipretide $40 \mathrm{mg} /$ day has been evaluated in patients aged $\geq 12$ years with Barth syndrome and in those aged $\geq 16$ years with primary mitochondrial myopathy, ${ }^{3,4}$ but there is very limited information on dosing for patients with other mitochondrial disorders, particularly for younger patients. In healthy adults and patients with primary mitochondrial myopathy, intravenous and subcutaneous doses of up to 0.25 $\mathrm{mg} / \mathrm{kg} / \mathrm{hr}$ (up to $140 \mathrm{mg} /$ day) for up to 7 days have been used in clinical investigations. PK analyses suggest that elamipretide shows dose-proportional exposure as measured by area under the plasma concentration-time curve (AUC) and maximum plasma concentrations at doses ranging from 2 to $80 \mathrm{mg}$. In adults, there was no apparent effect of age on the PKs of elamipretide although since elamipretide is eliminated renally, elamipretide exposure increases significantly as renal function decreases. In these instances, elamipretide was well tolerated with few systemic adverse events reported, primarily consisting of headache and dizziness. The most common adverse events are local injection-site reactions.

Dosing in younger patients in our case series assumed that the exposure-response relationship would be similar in young patients as for adolescents/adults. Therefore, the target dose of elamipretide for young patients was established by estimating the dose that would demonstrate comparable PK exposure (ie, AUC) to that seen with a $40 \mathrm{mg}$ dose in adults. Physiological-based PK modeling of pediatric physiology and its impact on the PKs of elamipretide was used to determine the dose. The results indicated that weight-normalized clearance in relation to blood flow does not differ significantly across the ages simulated. Therefore, plasma concentration-time profiles and AUC were expected to be similar for pediatric patients $<12$ years of age when utilizing weight-normalized doses. Based on our experience, a dose of approximately $0.5 \mathrm{mg} / \mathrm{kg} /$ day is well tolerated in pediatric patients aged $<12$ years of age. Alternatively, weight-based doses of 5,10 , and $20 \mathrm{mg} /$ day for patients weighing $<10,10-20$, and $20-40$ $\mathrm{kg}$, respectively, appear to be reasonable.

\section{Conclusions}

These cases provide evidence for the safe administration of weight-based dosing of elamipretide. This experience may guide the administration of elamipretide for future patients enrolled in the expanded access program.

\section{Methods}

Patients were treated under the expanded access Protocol: SPIES-006, an intermediate size expanded access protocol of elamipretide for subcutaneous injection in patients with genetically confirmed rare diseases with known mitochondrial dysfunction. Each subject received both FDA and local ethics review and approval prior to initiating therapy with elamipretide.

\section{Declarations}

Ethics Approval and consent to participate 
Patients enrolled in this study or their legal representative signed informed consent after review and approval by their individual institutions: UTHealth Committee for the Protection of Human Subjects HSCMS-20-0956 (IND 146005/137429), Landspitali Univerisy Icelandic Medicines Agency Lyfjastofnun \#030318-2770, Hospital of the University of Pennsylvania Institutional Review Board \#844643 (IND 153883), Nationwide Children's Hospital Institutional Review Board (IND 154167)

\section{Consent for publication}

Not applicable.

\section{Availability of data and materials}

Data sharing is not applicable to this article as no datasets were generated or analysed during the current study.

\section{Competing interests}

HTB is on a patent describing elamipretide for Sengers syndrome.

\section{Funding}

The authors wish to thank the staff at Stealth BioTherapeutics for providing drugs through their Expanded Access Program, and particularly the efforts and support of Anthony Abbruscato, Pharm D, and Donna Cowan, CCRC.

\section{Authors Contributions}

All authors participated in the data collection, data interpretation, and writing of these case reports. All authors had final responsibility for the decision to submit for publication.

\section{Acknowledgements}

The authors wish to thank the patients and their families for participating, as well as Bret Fulton, RPh, and Jim Shiffer, RPh, Write On Time Medical Communications, LLC, for their writing and editorial assistance during the development of this manuscript. Mary Kay Koenig wishes to thank her research nurse, Lindsey Miller, for providing patient support and assisting with administration and education responsibilities. Scott Falk would like to thank Dr. Marisa Cevasco and the cardiothoracic surgical and heart failure teams at the University of Pennsylvania, as well as Drs. Marni Falk and Theodore Drivas of the Mitochondrial Medicine Frontier Program, Division of Human Genetics, Department of Pediatrics, Children's Hospital of Philadelphia and University of Pennsylvania Perelman School of Medicine, Philadelphia, PA, USA.

\section{References}


1. Szeto HH. First-in-class cardiolipin-protective compound as a therapeutic agent to restore mitochondrial bioenergetics. Br J Pharmacol. 2014;171:2029-50.

2. Allen ME, Pennington ER, Perry JB, Dadoo S, Makrecka-Kuka M, Dambrova M, et al. The cardiolipinbinding peptide elamipretide mitigates fragmentation of cristae networks following cardiac ischemia reperfusion in rats. Commun Biol. 2020;3:389.

3. Thompson WR, Hornby B, Manuel R, Bradley E, Laux J, Carr J, et al. A phase 2/3 randomized clinical trial followed by an open-label extension to evaluate the effectiveness of Barth syndrome, a genetic disorder of mitochondrial cardiolipin metabolism. Genet Med. 2021 Mar;23(3):471-478.

4. Karaa A, Haas R, Goldstein A, Vockley J, Weaver WD, Cohen BH. Randomized dose-escalation trial of elamipretide in adults with primary mitochondrial myopathy. Neurology. 2018;90:e1212-21.

5. US Food and Drug Administration. Expanded access to investigational drugs for treatment usequestions and answers. Guidance for industry. 2017. https://www.fda.gov/media/85675/download. Accessed 22 May 2021.

6. Repp BM, Mastantuono E, Alston CL, Schiff M, Haack TB, Rötig A, et al. Clinical, biochemical and genetic spectrum of 70 patients with ACAD9 deficiency: is riboflavin supplementation effective? Orphanet J Rare Dis. 2018 Jul 19;13(1):120.

7. Bione S, D’Adamo P, Maestrini E, Gedeon AK, Bolhuis PA, Toniolo D. A novel X-linked gene, G4.5. is responsible for Barth syndrome. Nat Genet. 1996;12:385-9.

8. Clarke SL, Bowron A, Gonzalez IL, Groves SJ, Newbury-Ecob R, Clayton N, et al. Barth syndrome. Orphanet J Rare Dis. 2013;8:23.

9. Saric A, Andreau K, Armand A-S, Moller IM, Petit PX. Barth syndrome: from mitochondrial dysfunctions associated with aberrant production of reactive oxygen species to pluripotent stem cell studies. Front Genetics. 2016; doi:10.3389/fgene.2015.00359.

10. Lu Y-W, Claypool SM. Disorders of phospholipid metabolism: an emerging class of mitochondrial disease due to defects in nuclear genes. Front Genetics. 2015; doi:10.3389/fgene.2015.00003.

11. Vukotic M, Nolte H, König T, Saita S, Ananjew M, Krüger M, et al. Acylglycerol kinase mutated in Sengers syndrome is a subunit of the TIM22 protein translocase in mitochondria. Molecular Cell. 2017;67:471-3.

12. Kang Y, Stroud DA, Baker MJ, De Souza DP, Frazier AE, Liem M, et al. Sengers syndrome-associated mitochondrial acylglycerol kinase is a subunit of the human TIMM22 protein import complex. Molecular Cell. 2017;67:457-70.

13. El-Hattab AW, Scaglia F. Mitochondrial cardiomyopathies. Front Cardiovasc Med. 2016; doi:10.3389/fcvm.2016.00025.

14. He M, Rutledge SL, Kelly DR, Palmer CA, Murdoch G, Majumder N, et al. A new genetic disorder in mitochondrial fatty acid beta-oxidation: ACAD9 deficiency. Am J Hum Genet. 2007 Jul;81(1):87-103.

\section{Figures}




\section{Senger Syndrome}

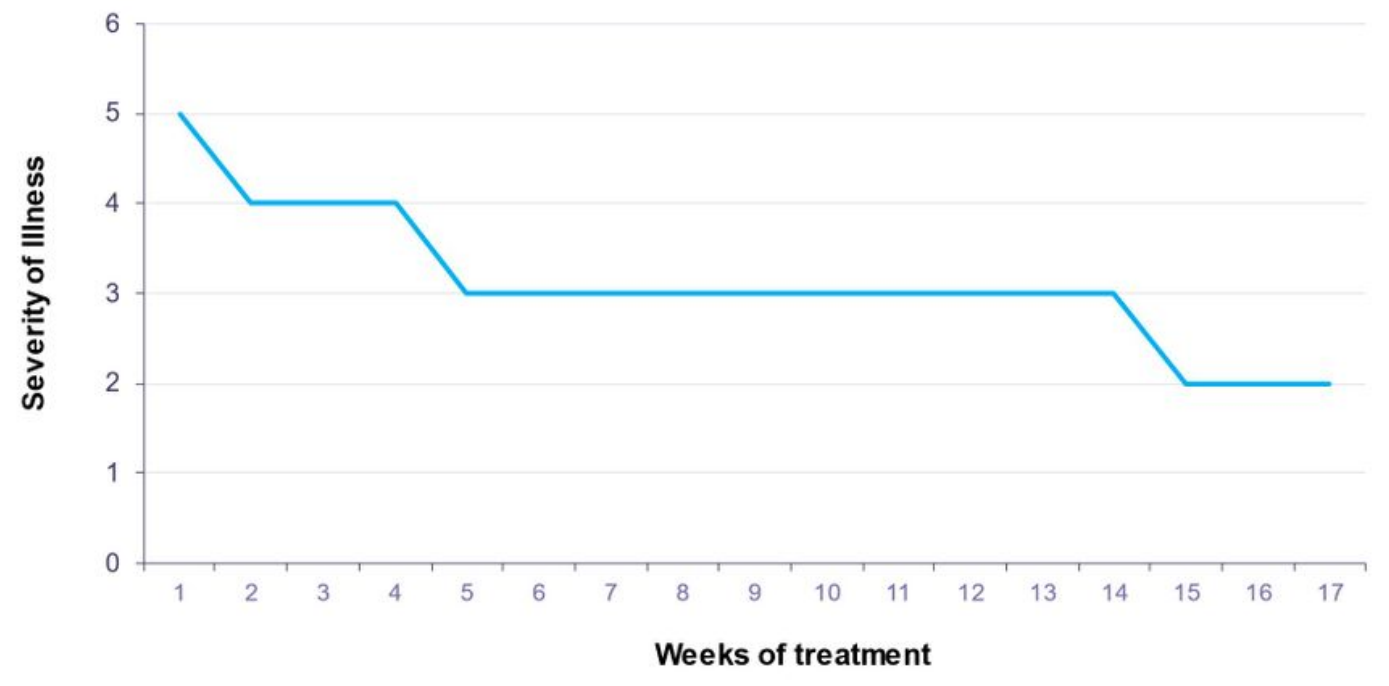

\section{Figure 1}

Severity score during the first 4 months of treatment with elamipretide 